\title{
Avaliação da Resistência à Corrosão de Compósitos Ferromagnéticos com Policloropreno ao Ensaio de Névoa Salina
}

\author{
Magali S. Pinho \\ Instituto de Pesquisas da Marinha, RJ \\ Mauro Z. Sebrão \\ Centro de Pesquisas de Energia Elétrica, $R J$ \\ Regina C. R. Nunes, Bluma G. Soares \\ Instituto de Macromoléculas, UFRJ
}

\begin{abstract}
Resumo: Este trabalho tem por objetivo avaliar a resistência à corrosão de composições elastoméricas à base de policloropreno $(\mathrm{CR})$ contendo uma carga ferromagnética, após exposição à câmara de névoa salina. $\mathrm{O}$ material utilizado no carregamento foi o ferrocarbonila (CI). Este ensaio foi importante, pois evidenciou o processo corrosivo em curto espaço de tempo, através da exposição do material a uma atmosfera bastante agressiva de cloreto de sódio $(\mathrm{NaCl})$, uma vez que este compósito deverá ser exposto à atmosfera marítima. A formação de uma camada superficial de óxido de ferro $\left(\mathrm{Fe}_{2} \mathrm{O}_{3}\right)$ no compósito de $\mathrm{CI}$ :CR foi caracterizada pela técnica de microscopia eletrônica de varredura (SEM) acoplada com detetor de raios-X (EDX).
\end{abstract}

Palavras-chave: Ferrocarbonila, policloropreno, névoa salina, corrosão.

\section{The Effect from the Exposure to Salt Spray on the Corrosive Process of Ferromagnetic Composites with Polychloroprene}

Abstract: The corrosive effect from the exposure to salt spray was studied on chloroprene rubber (CR) matrices containing a particulate ferromagnetic filler, viz. carbonyl-iron powder (CIP). The experiments in salt spray chamber are able to create an aggressive condition of sodium chloride atmosphere $(\mathrm{NaCl})$, being important to minimize the time of exposure of composites in comparison to the real conditions. The presence of corrosive products was visually observed at the surface of CI:CR composites (80:20 wt \%). The surface and interior of these composites were characterized by scanning electron microscopy (SEM), equipped with an energy-dispersive X-ray (EDX) analysis system. From these experiments, it was possible to suggest the formation of a surface layer of iron oxide $\left(\mathrm{Fe}_{2} \mathrm{O}_{3}\right)$. This compound was not detected inside the bulk.

Keywords: Carbonyl-iron, chloroprene rubber, salt spray exposure, corrosion.

\section{Introdução}

Ferrocarbonila (CI) consiste de um pó cinza com granulometria inferior a $10 \mu \mathrm{m}$ e com propriedades ferro-magnéticas, sendo bastante utilizado em componentes eletrônicos ${ }^{[1]}$.
O policloropreno (CR) é um elastômero com elevada resistência à chama, ao ozônio e à absorção de umidade, propiciando inúmeras aplicações para fins navais ${ }^{[2]}$.

Compósitos de CI:CR obtidos através da dispersão destas partículas ferromagnéticas, podem ser utilizados como materiais absorvedores de micro-ondas 
(RAM, "radar absorbing materials") dentre outras aplicações $^{[2]}$. A utilização desses materiais em ambiente marinho depende, entretanto, da sua resistência à exposição a essa atmosfera salina, bastante corrosiva.

O objetivo desse trabalho é, portanto, avaliar a resistência à corrosão desses compósitos utilizando um método eficiente e rápido, através da exposição à câmara de névoa salina. Tal método é capaz de proporcionar uma resposta rápida ao lento processo corrosivo que, geralmente ocorre nos componentes quando submetidos à atmosfera marítma.

A resistência à corrosão foi acompanhada através da técnica de microscopia eletrônica de varredura (SEM) acoplada com detector de raios-X (EDX). A utilização desse detector teve como objetivo confirmar a presença de uma camada superficial de óxido de ferro $\left(\mathrm{Fe}_{2} \mathrm{O}_{3}\right)^{[3]}$.

\section{Experimental}

\section{Materiais}

O ferrocarbonila (CI) do tipo EN, da BASF e a borracha de cloropreno, da Du Pont (CR, Neoprene $\mathrm{W}$, massa específica $\left.=1,2062 \mathrm{~g} / \mathrm{cm}^{3} ; \mathrm{M}_{\mathrm{w}}=440.000\right)$, além dos aditivos de vulcanização, foram utilizados como adquiridos.

\section{Caracterização do $\mathrm{Cl}$}

Os parâmetros magnéticos, tais como o campo coercitivo (Hc) e a magnetização de saturação (Ms), foram obtidos pelos ciclos de histerese, fornecidos pelo magnetrômetro de amostra vibrante (VSM) da EG\&G Princeton Applied Research, Mod. 4500.

A distribuição de tamanho de partículas foi determinada pelo sedígrafo $5100 \mathrm{~V} 3.07$ da Micromeritics ${ }^{[4]}$.

\section{Obtenção de Misturas com o CR}

As misturas físicas (blendas) com razões ponderais de 0:100, codificada como branco, 20:80, 50:50, 60:40 e 80:20 (\% em peso de CI:CR) foram realizadas em um misturador de cilindros Berstorff à temperatura ambiente e com velocidades de 22 e $25 \mathrm{rpm}$ (anterior e posterior), de acordo com os procedimentos clássicos empregados pela indústria de borracha. As blendas foram moldadas por compressão em uma prensa hidráulica a $150{ }^{\circ} \mathrm{C}$ e $6,7 \mathrm{MPa}$, de acordo com os tempos de vulcanização determinados pelo Reômetro de Disco Oscilatório Monsanto TM 100. Os corpos de prova foram obtidos sob a forma de tapetes vulcanizados, com as dimensões $(4,0 \times 4,0 \mathrm{~cm})$ e espessura de $0,10 \mathrm{~cm}$.

\section{Medidas de Condutividade Elétrica}

As medidas de condutividade elétrica superficial foram realizadas pelo método padrão de 4 pontas, através do emprego de um eletrômetro Keithley 617. Todas as medidas foram realizadas à temperatura ambiente com $50 \%$ de umidade relativa ${ }^{[5,6]}$.

\section{Estudo Morfológico}

A observação micromorfológica foi realizada nos tapetes vulcanizados, através do emprego de um microscópio eletrônico de varredura (SEM) Leica, modelo S440. A superfície criofraturada foi recoberta com ouro e o detetor utilizado foi o de elétrons secundários (SED).

As composições químicas da superfície e do interior da amostra de CI:CR (80:20) foram analisadas por SEM acoplada à Espectroscopia de Energia Dispersiva de Raios-X (EDX), utilizando o detetor de $\mathrm{QBSD}^{[3]}$.

\section{Ensaio de Resistência à Intempéries}

O teste realizado para a avaliação da resistência a intempéries consistiu do Ensaio de Exposição à Câmara de Névoa Salina Druckwal, modelo CA-680. Este ensaio foi realizado segundo a norma ASTM B-117-90, com o intuito de avaliar a resistência de materiais à corrosão, através da exposição à uma atmosfera salina de cloreto de sódio $(\mathrm{NaCl})$. O tempo deste ensaio foi de aproximadamente 744 horas, equivalente a $31 \operatorname{dias}^{[7]}$.

Após exposição dos materiais à câmara de névoa salina, os corpos de prova foram lavados com água destilada, para remover o excesso de cloreto de sódio remanescente, com posterior secagem em estufa a $80{ }^{\circ} \mathrm{C}$ por $48 \mathrm{~h}$.

\section{Resultados e Discussão}

Pela histeresimetria magnética o CI apresentouse como um material magneticamente mole, com campo coercitivo de $356 \mathrm{Oe}^{[8]}$.

A distribuição de tamanho de partículas referente à amostra de ferrocarbonila foi obtida a partir de análise em sedígrafo, cujo resultado encontra-se na Figura 1. Observa-se que, cerca de $96 \%$ da massa 


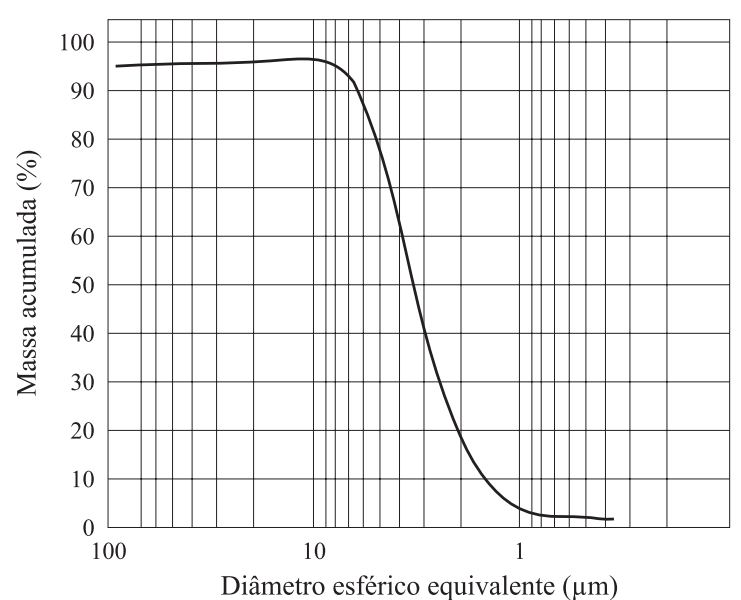

Figura 1. Massa acumulada (\%) versus diâmetro esférico equivalente $(\mu \mathrm{m})$ para a amostra de CI.

acumulada total possui diâmetro de partícula na faixa de 1 a $9 \mu \mathrm{m}$, com diâmetro médio de $3,35 \pm 0,01 \mu \mathrm{m} \mathrm{e}$ diâmetro modal de 3,67 $\pm 0,01 \mu \mathrm{m}$.

A Tabela 1 ilustra os valores de condutividade elétrica superficial para as diferentes composições percentuais em peso de CI:CR. Torna-se relevante mencionar que a composição isenta de borracha (100:0) foi prensada sob a forma de pó. Embora o CI apresente valor elevado de condutividade elétrica, os compósitos com policloropreno resultaram em materiais isolantes, mesmo quando teores tão altos como $80 \%$ de ferrocarbonila foram adicionados à matriz elastomérica. Estes resultados indicam que o aditivo condutor não é capaz de formar os caminhos condutores, ou seja, um sistema percolativo mesmo em altas concentrações. Acredita-se que a pequena área específica $\left(0,9859 \mathrm{~m}^{2} / \mathrm{g}\right)$, associada à forma esférica deste aditivo, sejam responsáveis pelo comportamento isolante.

A análise micromorfológica apresentada na Figura 2 referente à composição 80:20 de CI:CR (\%

Tabela 1. Condutividade superficial para as composições percentuais em peso de CI:CR.

\begin{tabular}{cc}
\hline $\begin{array}{c}\text { Composição de CI:CR } \\
(\% \text { peso })\end{array}$ & $\begin{array}{c}\text { Condutividade Superficial } \\
(\boldsymbol{\sigma}, \mathbf{S} / \mathbf{c m})\end{array}$ \\
\hline $0: 100$ & $5,5 \times 10^{-12}$ \\
$20: 80$ & $3,8 \times 10^{-11}$ \\
$50: 50$ & $5,0 \times 10^{-11}$ \\
$60: 40$ & $5,8 \times 10^{-11}$ \\
$80: 20$ & $9,0 \times 10^{-11}$ \\
$100: 0$ & $4,4 \times 10^{2}$ \\
\hline
\end{tabular}

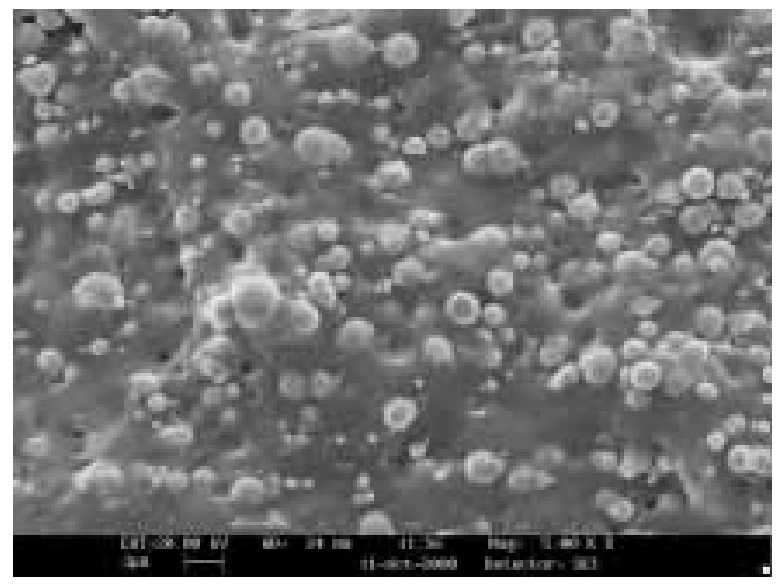

Figura 2. Microscopia eletrônica de varredura obtida pela fratura crioscópica da composição 80:20 de CI:CR.

em peso), confirma a ausência de caminho condutor nesse sistema. As partículas de ferrocarbonila apresentam uma morfologia esférica e uma dispersão bem homogênea na matriz de CR, apesar do elevado carregamento de $\mathrm{CI}$ empregado. As micrografias relacionadas aos compósitos contendo menores teores de CI não são apresentadas uma vez que se comportaram de forma semelhante.

Apesar da ausência de condutividade elétrica, ou seja, do seu caráter dielétrico, o compósito contendo $80 \%$ do aditivo apresentou elevada absorção de microondas na região entre 8 e $10 \mathrm{GHz}^{[2]}$, sugerindo uma aplicação em potencial como RAM.

Os compósitos contendo diferentes teores de CI foram expostos à névoa salina com o objetivo de verificar sua resistência à atmosfera marítima, requisito importante para serem utilizados em aplicações navais. A Figura 3 ilustra o aspecto visual observado para as diferentes composições percentuais em peso de CI:CR, após esse ensaio.

As composições percentuais em peso de CI:CR correspondentes à 20:80 e 50:50 apresentaram aspecto semelhante àquele observado para a composição 60:40, representada na Figura 3b. No entanto, a composição 80:20 apresentou um intenso processo corrosivo, visível a olho nu, por toda a extensão da amostra conforme ilustra a Figura 3c.

A confirmação da presença superficial de óxido de ferro foi possível através de microscopia eletrônica de varredura acoplada a EDX. A Figura 4a (lado esquerdo da amostra) ilustra a imagem correspondente ao interior da amostra, obtida a partir de fratura crioscópica do material. A imagem ilustrada pela Figura $4 \mathrm{~b}$ (lado direito) corresponde à superfície da 


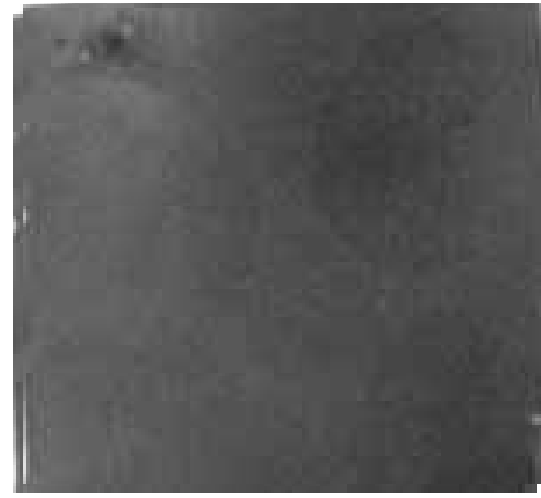

(a)

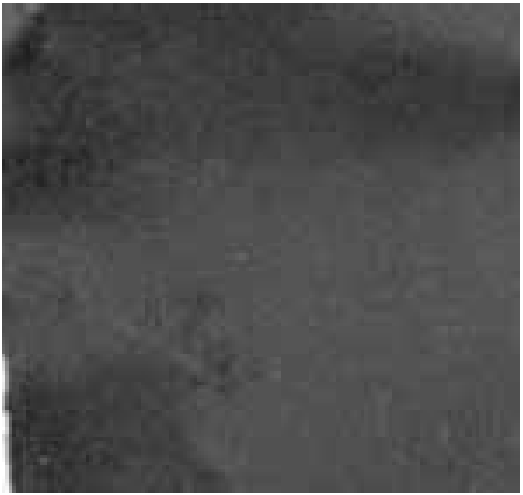

(b)

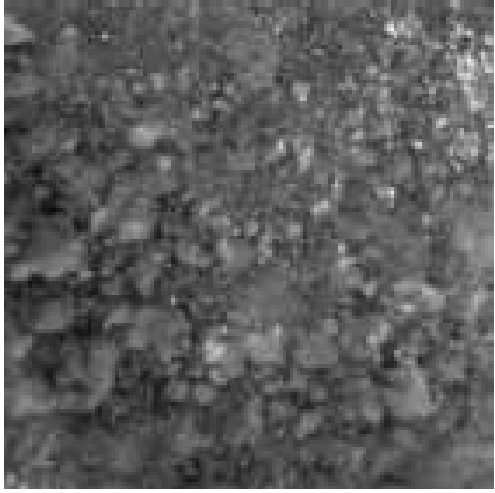

(c)

Figura 3. Aspecto observado após exposição à névoa salina para as composições de CI:CR, (a) 0:100; (b) 60:40 e (c) 80:20.

mesma. Observam-se diferenças morfológicas significativas entre a superfície e o interior da amostra.

A composição química da superfície da amostra (Figuras $4 \mathrm{~b}$ e $4 \mathrm{c}$ ) foi analisada pela técnica de EDX, cujos espectros referentes à microanálise química pontual são ilustrados na Figura 5.

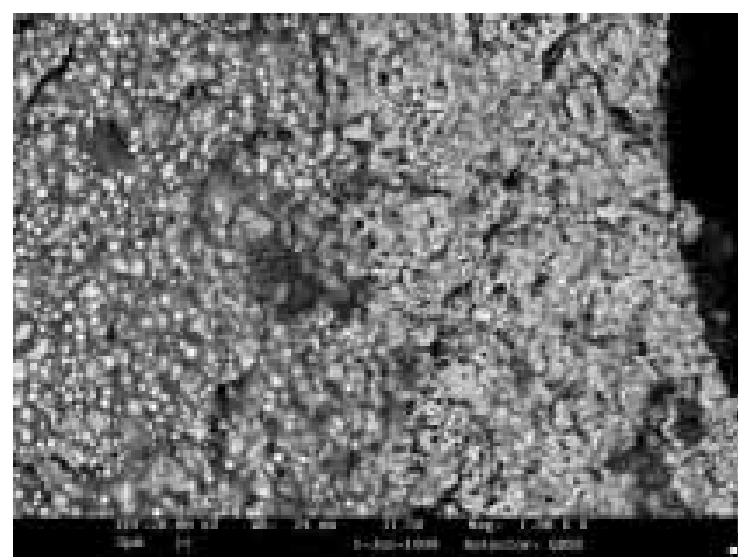

(a)

(b)

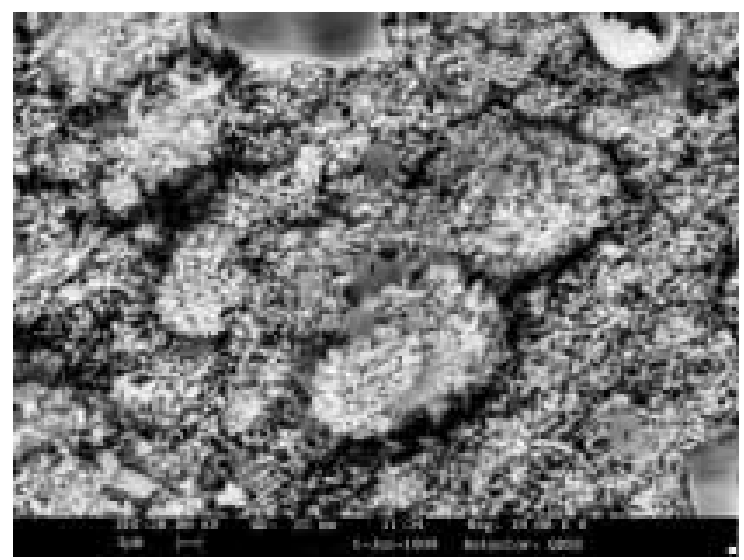

(c)

Figura 4. Microscopias eletrônicas de varredura para a composição percentual em peso 80:20 de CI:CR, (a) interior (fratura crioscópica) e (b) superfície da amostra, constituída por (c) cristais de $\mathrm{NaCl}$ (1) e $\mathrm{Fe}_{2} \mathrm{O}_{3}{ }^{[2]}$.
Os resultados apresentados nas Figuras 4 e 5 revelam, em primeiro lugar que a superfície da amostra (Figuras 4b, 4c e 5) apresenta-se de forma bem distinta do interior da mesma (Figura 4a) e que esta contém cristais esféricos de $\mathrm{NaCl}$, remanescentes do processo de lavagem (Figuras 4c e 5a) e cristais de óxido de ferro (Figuras $4 \mathrm{c}$ e $5 \mathrm{~b}$ ), sob a forma de ouriços. A

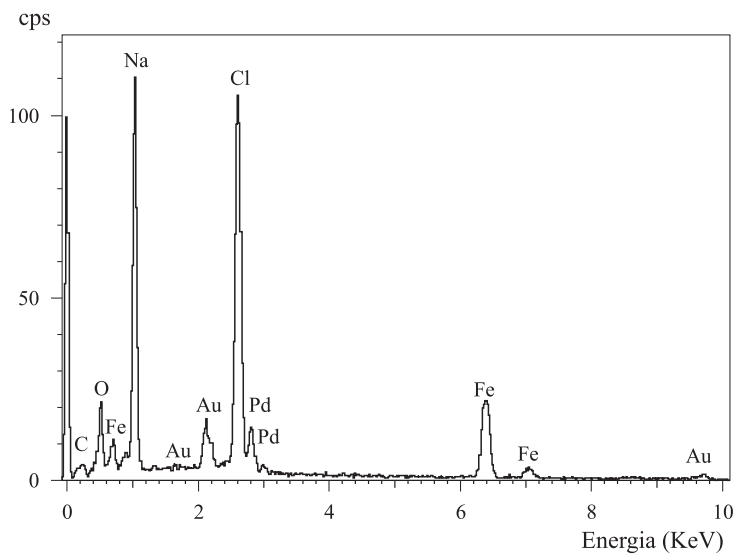

(a)

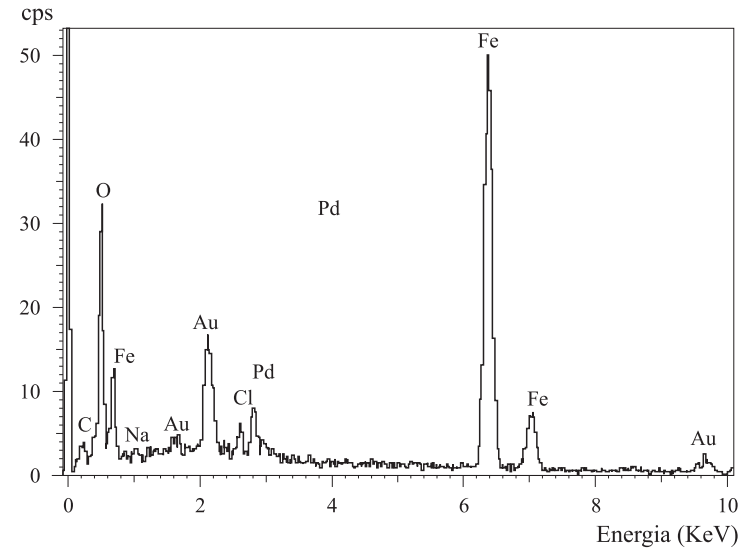

(b)

Figura 5. Espectros de EDX identificando a presença de (a) cristais de $\mathrm{NaCl}$ e (b) de $\mathrm{Fe}_{2} \mathrm{O}_{3}$, encontrados na superfície da amostra. 


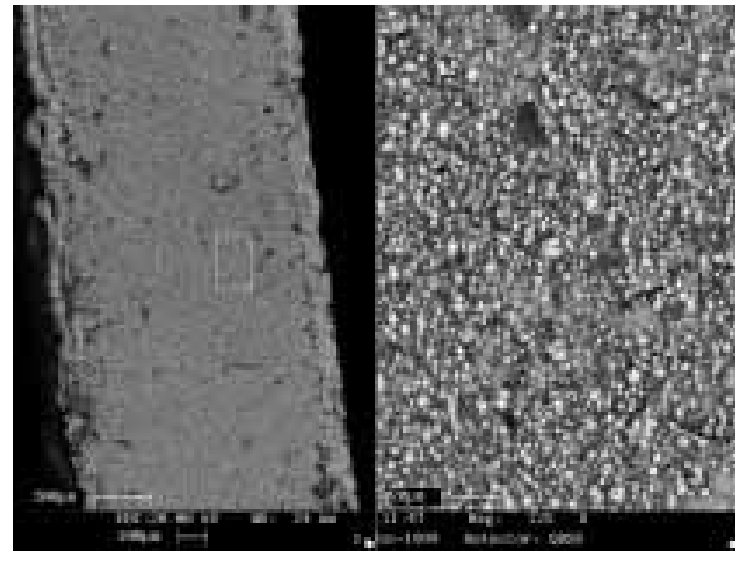

(a)

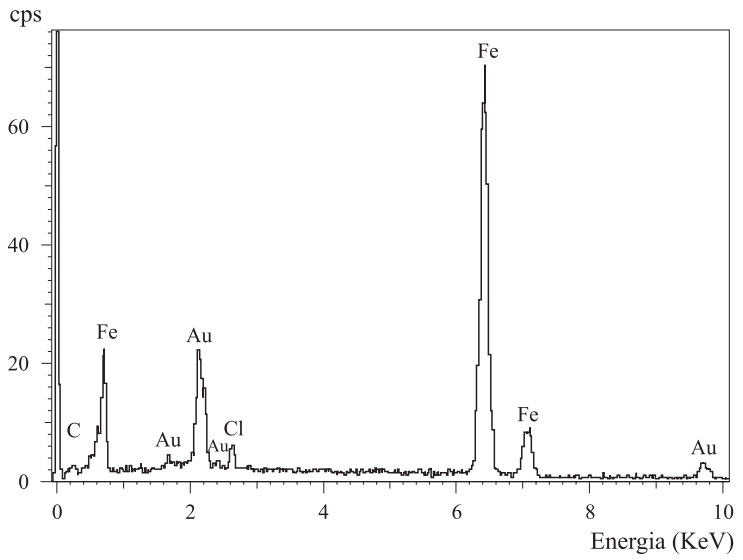

(b)

Figura 6. Microscopias eletrônicas de varredura (a) de CI:CR (80:20) e (b) espectro de EDX do interior da amostra, obtidos pela fratura crioscópica, após o ensaio de névoa salina.

presença destes cristais pode ser melhor visualizada na Figura 4c.

Com o objetivo de se avaliar a extensão da formação de óxido de ferro, após exposição à névoa salina, $o$ interior da amostra foi analisado por SEM e por EDX, através de fratura crioscópica, conforme ilustra a Figura 6.

As imagens apresentadas na Figura 6 indicam a presença de partículas esféricas atribuídas à CI, corroborando a imagem apresentada na Figura 2. Comparando-se os espectros de EDX obtidos a partir da análise da superfície da amostra (Figura 5) com aquele do interior da mesma (Figura 6b), pode-se afirmar que os compostos de ferro são diferentes. No espectro da Figura 5b, observa-se um pico bastante intenso relativo ao oxigênio, devido à presença do óxido de ferro. No espectro correspondente ao interior da amostra (Figura 6b), este pico não foi detectado, indicando que o composto de ferro, localizado no interior da mesma, refere-se ao ferrocarbonila, cujo espectro de EDX é

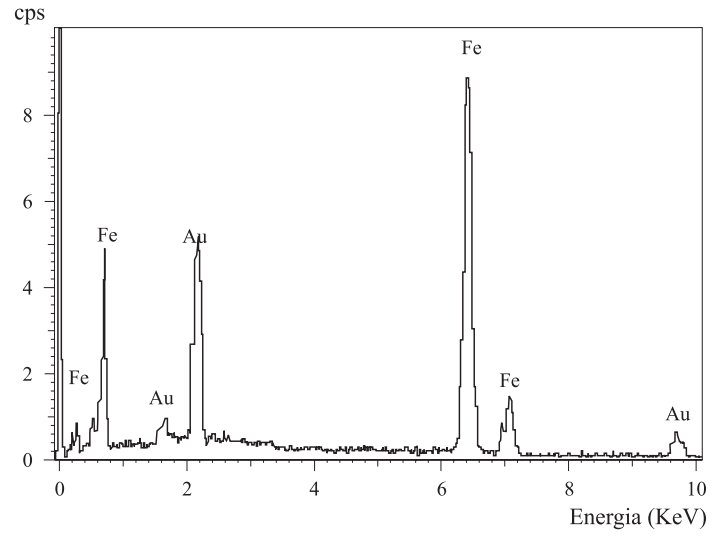

Figura 7: Espectro de EDX do CI.

apresentado na Figura 7. Além desses picos, observase também a presença de outros picos, referentes aos elementos químicos $\mathrm{Au}$ (ouro) e Pd (paládio), decorrentes do processo de metalização e do elemento $\mathrm{Cl}$, oriundo do policloropreno.

\section{Conclusões}

De forma diferente das demais composições percentuais em peso de CI:CR, a 80:20 não apresentou uma boa resistência à corrosão após exposição à névoa salina, tornando-se necessário o emprego de um recobrimento anti corrosivo.

Através do emprego da Microscopia Eletrônica de Varredura (SEM) acoplada com detector de raios$\mathrm{X}$ (EDX), foi possível concluir que o processo corrosivo só ocorreu a nível superficial, pois através da análise da imagem obtida pela fratura crioscópica não foi observada a presença de óxido de ferro no interior da amostra, tendo sido detectada apenas a presença do elemento ferro, pertinente ao ferrocarbonila. Apesar do compósito contendo $80 \%$ de ferrocarbonila apresentar um processo corrosivo acentuado, o seu desempenho como um RAM para a faixa de 8 a $10 \mathrm{GHz}$ não sofreu alterações significativas, como publicado anteriormente ${ }^{[2]}$.

\section{Agradecimentos}

Os autores agradecem ao Dr. Arnaldo Alcover Neto do Centro de Tecnologia Mineral (CETEM) pelas análises de SEM.

\section{Referências Bibliográficas}

1. Smith, F. C.; Chambers, B. \& Bennett, J. C. - IEE Proc. Sci. Meas. Technol., 141, p.538 (1994). 
2. Pinho, M. S.; Gregori, M. A.; Nunes, R. C. \& Soares, B. G. - Polym. Degrad. Stab.,73, p.1 (2001).

3. Fan, J.; Huang, B.; Qu, X. \& Zou, Z. - International Journal of Refratory. Metals and Hard Materials, 19, p.73 (2001).

4. Washington, C. - "Particle Size Analysis in Pharmaceutics and other Industries", Ellis Horwood Limited, New York (1992).

5. Lima, E. V.; Wood, P. E.; Hamielec, A. E. \& Penlidis A. - Ind. Eng. Chem. Res., 36, p.939 (1997).
6. Pinho, M. S.; Gorelova, M. M.; Dezzoti, M. \& Soares, B. G. - J. Appl. Polym. Sci., 70, p. 1543 (1998).

7. American Society for Testing and Materials, vol. 02.05, Philadelphia, 1990- Método ASTM B 117-90, "Standard Test Method of Salt Spray (Fog) Testing".

8. Praveen, S.; Babbar, V. K.; Razdan, A.; Puri, R. K. \& Goel T. C. - J. Applied Physics, 87, p. 9 (2000).

Recebido: $30 / 10 / 01$

Aprovado: 30/10/02 